influenza-like symptoms, said the CDC in the November 10, 2000, issue of the Morbidity and Mortality Weekly Report. CM is a fungal disease endemic in the southwestern United States and parts of Central and South America. The disease is acquired by inhaling the spores of Coccidioides immitis, present in soil. Symptoms are usually mild and influenza-like.

Twenty-seven of 35 church members became ill last winter within 2 weeks after returning from Hermosillo, Mexico, where they had spent a week building a church. Initial testing of acute serum specimens by the CDC showed that 1 member had antibodies to $C$ immitis. Subsequently, serum samples and questionnaires were obtained for 30 of the 35 travelers. Of these, 23 reported they had gotten sick in Mexico or after returning home. Serological testing indicated that 8 people, 7 of whom were symptomatic, met the case definition for $\mathrm{CM}$. The most common symptoms were fatigue, fever, and joint and muscle pain; 3 people had a rash, and 4 had a cough. One traveler was hospitalized for a day, and 11 missed work or school (an average of 5.5 days). Eighteen people sought treatment for the illness, and 11 received medications, such as fluconazole or itraconazole.

No activities other than the travel and construction work were associated with the illness. Twenty-two of the church members reported working in very dusty conditions. Approximately $40 \%$ of persons infected with $C$ immi$t i s$ experience symptoms, usually mild and influenza-like; however, $8 \%$ of patients may develop severe pulmonary disease requiring hospitalization, and $7 \%$ develop disseminated extrapulmonary disease. Risk factors for severe pulmonary disease include diabetes, smoking, and older age, whereas risk factors for disseminated disease include black or Asian race, pregnancy, and immunocompromising conditions.

Although avoiding activities that generate dust or using a mask during these activities is advisable, these measures do not provide complete protection from CM. A potential strategy for adequate prevention is vaccine development, because natural infection with $C$ immitis provides lifelong immunity. However, until a vaccine becomes available, organizations that conduct trips to areas where $\mathrm{CM}$ is endemic should inform their travelers about the risks for $\mathrm{CM}$. Healthcare providers should consider CM in travelers returning from areas where the disease is endemic and who present with an influenza-like illness.

FROM: icanNEWS. November 10, 2000.

\section{Community-Acquired MRSA in Queensland, Australia}

Community-acquired methicillin-resistant Staphylococcus aureus (MRSA) susceptible to gentamicin has been reported in a number of countries in the 1990s. To study the acquisition of gentamicin-sensitive (GS) MRSA in southeast Queensland and the relatedness of GS MRSA to other strains of MRSA, 35 cases of infection due to GS MRSA from October 1997 through September 1998 were examined retrospectively to determine the mode of acquisition and risk factors for MRSA acquisition.

Thirty-one isolates from the cases were examined using a variety of methods (antibiotyping, phage typing, pulsed-field gel electrophoresis [PFGE] fingerprinting, and coagulase typing by restriction analysis of polymerase chain reaction products) and were compared with strains of local hospital-acquired GS MRSA and of Western Australian (WA) MRSA. Only 6 of 23 cases of communityacquired GS MRSA had risk factors for MRSA acquisition. Twenty of 21 isolates from cases of community-acquired infection were found to be related by PFGE and coagulase typing and had similar phage-typing patterns. Hospital- and nursing home-acquired GS MRSA strains were genetically and phenotypically diverse. Community-acquired GS MRSA strains were not related to nosocomial GR MRSA or WA MRSA, but phage-typing results suggest they are related to GS MRSA previously reported in New Zealand.

FROM: Nimmo GR, Schooneveldt J, O'Kane G, McCall B, Vickery A. Community acquisition of gentamicinsensitive methicillin-resistant Staphylococcus aureus in southeast Queensland, Australia. J Clin Microbiol 2000;38: 3926-3931.

\section{On-Line Lectures With International Focus}

\section{Judene Bartley}

The Supercourse: Epidemiology, the Internet, and Global Health web site offers a series of lectures on epidemiology and the Internet in English, Spanish, and Japanese for students of medicine and health. The British Medical Journal describes the course as the Global Health Network University. The Supercourse has 1,700 faculty from 110 countries who created a Library of Lectures, with 214 lectures currently available.

The lectures are grouped under the headings New, Revised; Epidemiology and Biostatistics; and Internet and Global Health. Examples of topics include "A Brief Introduction to Epidemiology," "Antibiotics Use and Medical Drug Policy," "Epidemiology of Disasters," "Introduction to Program Evaluation," "Environmental Epidemiology," and "Evidence-Based Medicine: Can We Practice Effectively Without it?" Lectures on specific diseases include, but are not limited to, malaria, cancer, diabetes, and hepatitis. After linking to the index, viewers may simply click on topics and move through the slides and text at their own pace. Hyperlinks within slides permit instant access to sites with related, in-depth information. Interested parties are welcomed to join and either share or review lectures on preferred topics. Go to http:// www.pitt.edu/ super1/. 